\title{
Investigating the Solid-Liquid Phase Transition of Water Nanofilms Using the Generalized Replica Exchange Method
}

\author{
Qing Lu, ${ }^{1}$ Jaegil Kim, ${ }^{2, *}$ James D. Farrell, ${ }^{2}$ David J. Wales, ${ }^{2}$ and John E. Straub ${ }^{3, \dagger}$ \\ ${ }^{1}$ Division of Materials Science and Engineering, \\ Boston University, Brookline, MA 02446, USA. \\ ${ }^{2}$ University Chemical Laboratories, Lensfield Road, \\ Cambridge CB2 1EW, United Kingdom \\ ${ }^{3}$ Department of Chemistry, Boston University, Boston, MA, 02215 \\ (Received)
}

\begin{abstract}
The Generalized Replica Exchange Method (gREM) was applied to simulate a solid-liquid phase transition in a nanoconfined bilayer water system using the monatomic water $(\mathrm{mW})$ model. Merging an optimally designed non-Boltzmann sampling weight with replica exchange, gREM is particularly well suited for the effective simulation of first-order phase transitions characterized by S-bends ("backbending") in the statistical temperature and a bimodal structure in the canonical probability density function. The effective temperatures of gREM were designed to form unique crossing points with the statistical temperature, thereby facilitating sampling of energy states across the transition region. Statistical Temperature Weighted Histogram Analysis Method (ST-WHAM) was used to reweight gREM simulation results into canonical ensemble averages, including the Helmholtz free energy, internal energy, and heat capacity. The minimized structures of bilayer water systems with varying sizes were obtained through basin-hopping global optimization using the GMIN package, and ice structures composed of pentagons, hexagons and heptagons were observed.
\end{abstract}

${ }^{*}$ Current address: Broad Institute of MIT and Harvard, Cambridge, MA 02142, USA.

${ }^{\dagger}$ Author to whom correspondence should be addressed. Electronic mail: straub@bu.edu 


\section{INTRODUCTION}

The phase behavior of bulk water has received extensive interest due to the rich complexity of structures characterizing liquid, solid and clusters [1-4]. Nanoscale confined water adds a new dimension of phase behavior and has generated intense interest [5-10] due to its relevance in biology and materials science. In the case of water confined between two infinite parallel plates, monolayer ice, bilayer ice, and three-layer ice structures have been observed as a function of the separation between the plates $[7,11,12]$. Bilayer water can form various crystal, quasicrystals, and amorphous structures, including hexagonal ice, pure pentagonal ice, mixed hexagonal and pentagonal ice, and dodecagonal quasicrystals [8]. The transitions from liquid to various crystal and quasicrystal states were shown to be first-order, based on the sharp drop in the potential energy and discontinuity in the diffusion coefficient.

First-order phase transitions have a unique feature in the statistical temperature referred to as an S-bend, through which the temperature decreases upon absorbing energy in the region of metastable and unstable states. The behavior results from the depletion of phase-coexistent configurations associated with the free energy penalty for forming interfaces [13]. These features are associated with a bimodal structure in the energy distribution, the indicator of two-phase coexistence, in which the energy states between the two peaks are intrinsically unstable for the canonical ensemble [14-18]. A natural way to enhance sampling in the phase-coexistence region is the replacement of canonical sampling in temperature with non-canonical distributions. The generalized Replica Exchange Method (gREM) [19] incorporates a non-Boltzmann sampling weight from a generalized ensemble into the replica exchange paradigm [20-32]. The generalized ensemble sampling weights are determined from tailored effective temperatures through an inverse mapping strategy. The mapping is equivalent to umbrella sampling for a number of energy windows, with a "thermometer" in each window. Since its development, gREM has been applied to study phase transitions in Potts spin systems, an adapted Dzutugov model, Lennard-Jones fluid, and bulk water [19, 33-35]. Here we study the solid-liquid phase transition in bilayer confined water as a demonstration of the utility of the generalized Replica Exchange Method. 


\section{METHODS AND MATERIALS}

\section{A. Generalized Exchange Method}

In a gREM simulation there are $M$ replicas. Each replica $\alpha,(\alpha=1, \cdots, M)$, is assigned an effective temperature $T_{\alpha}\left(E ; \lambda_{\alpha}\right)$ and samples energy states consistent with the generalized ensemble weight $W_{\alpha}\left(E ; \lambda_{\alpha}\right)$. The sampling weight $W_{\alpha}\left(E, \lambda_{\alpha}\right)$ is determined from the effective temperature through the inverse mapping strategy

$$
T_{\alpha}\left(E ; \lambda_{\alpha}\right)=\left[\partial w_{\alpha} / \partial E\right]^{-1}
$$

where $w_{\alpha}=-\ln W_{\alpha}$ is the generalized effective potential.

The effective temperature is conveniently parameterized using linear functions with uniform slope for all replicas $(\alpha=1, \cdots, M)$ as

$$
T_{\alpha}\left(E ; \lambda_{\alpha}\right)=\lambda_{\alpha}+\gamma_{0}\left(E-E_{0}\right)
$$

where the control parameter $\gamma_{0}$ is the constant slope, $E_{0}$ is a constant in the relevant energy range, and $\lambda_{\alpha}$ is the $T$-intercept at a chosen $E_{0}$.

The linear effective temperature of Eq. (2) produces a sampling weight

$$
W_{\alpha}\left(E ; \lambda_{\alpha}\right) \sim\left[\lambda_{\alpha}+\gamma_{0}\left(E-E_{0}\right)\right]^{-1 / \gamma_{0}}
$$

The sampling weight in Eq. (3) governs the trial moves within one replica and replica exchanges between neighboring replicas. The acceptance ratio of a Monte Carlo trial move in configuration space within replica $\alpha$ is

$$
A_{\mathrm{gREM}}\left(\mathbf{x} \rightarrow \mathbf{x}^{\prime}\right)=\min \left[1, e^{w_{\alpha}(E(\mathbf{x}))-w_{\alpha}\left(E\left(\mathbf{x}^{\prime}\right)\right)}\right]
$$

The acceptance ratio for replica exchange between neighboring replicas $\alpha$ and $\alpha+1$ is

$$
A_{\mathrm{gREM}}\left(\alpha ; \mathbf{x} \mathbf{x}^{\prime}\right)=\min \left[1, \exp \left(\Delta_{\alpha}^{x}\right)\right]
$$

where $\Delta_{\alpha}^{\mathbf{x}}=w_{\alpha+1}\left(E\left(\mathbf{x}^{\prime}\right)\right)-w_{\alpha+1}(E(\mathbf{x}))+w_{\alpha}(E(\mathbf{x}))-w_{\alpha}\left(E\left(\mathbf{x}^{\prime}\right)\right)$, and $w_{\alpha}=-\ln _{\alpha}$.

\section{B. Statistical Temperature WHAM}

As each replica $\alpha=1, \ldots, M$ in gREM samples non-canonical sampling weights $W_{\alpha}(E)$, the data must be reweighted to estimate canonical averages. We calculated the density of states $\Omega(E)$ and 
the entropy $S(E)=k_{B} \ln \Omega(E)$ by combining multiple generalized ensemble runs via the Statistical Temperature Weighted Histogram Analysis Method (ST-WHAM) [36-38]. Once the density of states is known, canonical thermodynamic averages can be determined for any temperature by the reweighting technique.

Unlike the conventional Weighted Histogram Analysis Method (WHAM) [39], ST-WHAM does not use an iterative technique to determine the relevant partition function, but instead determines the inverse statistical temperature, $\beta_{S}=1 / T_{S}$, directly from $W_{\alpha}(E)$ and the associated histogram $H_{\alpha}(E)$. The integration of $\beta_{S}=\partial \ln \tilde{\Omega}(E) / \partial E$ provides an estimate for the entropy and the density of states. This procedure leads to a substantial acceleration of the data analysis without loss in accuracy.

The ST-WHAM estimate for the inverse statistical temperature is

$$
\beta_{S}^{*}=\sum_{\alpha} f_{\alpha}^{*}(E)\left(\frac{\partial \ln H_{\alpha}}{\partial E}-\frac{\partial \ln W_{\alpha}}{\partial E}\right)
$$

where $H_{\alpha}(E)$ is the energy histogram in replica $\alpha, f_{\alpha}^{*}(E)=H_{\alpha}(E) / \sum_{\alpha} H_{\alpha}(E)$ is the simulated histogram fraction.

Integration of $\beta_{S}^{*}$ provides an entropy estimate $S(E)$ as well as $\Omega(E)$, but direct integration is not desirable due to the rapid variation of $\beta_{S}$ for small $E$. The statistical temperature $T_{S}^{*}$ was approximated on an equally spaced enthalpy grid which makes possible analytical integration [36]. Once $S(E)$ is determined, all canonical thermodynamic properties are completely determined.

The Helmholtz free energy at a given temperature $T$ is calculated by $F_{T}(E)=E-T S(E)$. The reweighted probability density function at $T$ is given by $P_{T}(E)=e^{-F_{T}(E) / T}=e^{S(E)-E / T}$. The canonical expectation value for any variable may then be computed as

$$
\langle A(T)\rangle=\frac{\int d E e^{S(E)-\beta E} A(E)}{\int d E e^{S(E)-\beta E}},
$$

and the canonical heat capacity is estimated through calculation of the fluctuations of the internal energy,

$$
C_{v}(T)=\frac{\left\langle E(T)^{2}\right\rangle-\langle E(T)\rangle^{2}}{k_{B} T^{2}}
$$

\section{Monatomic water $(\mathrm{mW})$ model}

We employed the monatomic water model $(\mathrm{mW})$, which is a coarse-grained model that represents a water molecule as an intermediate element between carbon and silicon [40]. This potential 
reproduces the structural, thermodynamic, and dynamic properties of liquid water with comparable or better accuracy than the most popular atomistic water models at much less computational cost. $\mathrm{mW}$ has been applied in the study of pure bulk water [41-43] and nanoconfined water [44, 45], as well as biological water [46] and clathrate hydrates [47].

In this study, the water-like molecules were confined between two smooth hydrophobic walls at a fixed distance of $8.5 \AA$. The water-wall interaction was governed by a Lennard-Jones 9-3 potential with $\sigma=3.56 \AA$ and $\epsilon=0.569 \mathrm{~kJ} \mathrm{~mol}^{-1}$ [7]. We simulated systems composed of $N=256$ water molecules confined between two parallel plates of size $L \times L$, where $L$ is a length varying between 31.2 and $35.8 \AA$ A. Periodic boundary conditions were applied in lateral directions. 32 replicas were used in gREM simulations to cover temperature from $T_{1}=200 \mathrm{~K}$ to $T_{32}=320 \mathrm{~K}$.

\section{Water structure analysis}

We calculated the lateral radial distribution function (RDF) $g_{x y}(r)$ versus the lateral position $r_{x y}$ parallel to the confining plates. The lateral RDF is defined by [11]

$$
g_{x y}(r)=\frac{1}{\rho^{2} V} \sum_{i \neq j} \delta\left(r-r_{i j}\right)\left[\theta\left(\left|z_{i}-z_{j}\right|+\delta z / 2\right)-\theta\left(\left|z_{i}-z_{j}\right|-\delta z / 2\right)\right],
$$

where $V$ is the volume, $r_{i j}$ is the lateral distance between coarse-grained molecules $i$ and $j, z$ is the $z$ coordinate, and $\delta(x)$ is the Dirac $\delta$ function. The Heaviside function $\theta(x)$ restricts the sum to pairs within the same layer.

The lateral static structure factor $S(q)$ is the Fourier transform of the lateral radial distribution function $g_{x y}(r)[48,49]$ according to

$$
S(Q)=1+2 \pi \rho \int_{0}^{L} r\left(\frac{\sin (q r)}{q r}\right)[g(r)-1] d r .
$$

The wave vector $q$ is defined as $q=2 \pi k / L$, where $k$ is an integer that ranges from 1 to $N$, the total number of water molecules, and $L$ is the length of the simulation box.

\section{E. Basin-hopping global optimization}

Basin-hopping (BH) global optimization [50, 51], as implemented in the GMIN [52] package, was used to explore the potential energy landscape. The BH scheme used in this work is as follows:

1. a random Cartesian displacement is applied to the initial coordinates, $\boldsymbol{r}_{i}$; 
2. the perturbed coordinates, $\boldsymbol{r}_{i}^{\prime}$, are quenched to the local minimum, $\boldsymbol{r}_{n}$;

3. the new configuration, $\boldsymbol{r}_{n}$, is accepted with probability

$p(i \rightarrow n)=\min \left(1, e^{-\beta \Delta E}\right)$

where $\Delta=E_{n}-E_{i}, E_{i}$ and $E_{n}$ are the energies of the initial and new configurations, and $\beta=1 / k T$.

$6 \times 10^{3} \mathrm{BH}$ steps were run for each starting structure. At each step, random Cartesian displacements up to $0.8 \AA$ were applied to each particle. The temperature parameter $T$ was fixed at $8.0 \mathrm{~kJ} \mathrm{~mol}^{-1}$. Local optimization was performed using a modified version of Nocedal's limited memory BFGS (L-BFGS) minimizer[53, 54]. The root-mean-square gradient of the local minima was converged to $10^{-4} \mathrm{~kJ} \mathrm{~mol}^{-1} \AA^{-1}$.

\section{RESULTS AND DISCUSSION}

gREM utilizes optimally designed effective temperatures $T_{\alpha}(E)$ ensuring that unstable or metastable energy states of the canonical ensemble in the S-bend region are transformed into stable states having a unimodal probability distribution function (PDF).

To implement gREM, a necessary and sufficient condition on $T_{\alpha}\left(E ; \lambda_{\alpha}\right)$ is derived by identifying an extremum, $E_{\alpha}^{*}$, of a generalized free energy density, $\beta \mathcal{F}_{\alpha}(E)=w_{\alpha}(E)-S(E)$, as

$$
T_{\alpha}\left(E_{\alpha}^{*} ; \xi_{\alpha}\right)=T_{S}\left(E_{\alpha}^{*}\right)=T_{\alpha}^{*}
$$

where $T_{S}(E)=[\partial S / \partial E]^{-1}$ is the statistical temperature and $E_{\alpha}^{*}$ is the crossing point between $T_{\alpha}(E)$ and $T_{S}(E)$. The stability condition

$$
\beta \mathcal{F}_{\alpha}^{\prime \prime}\left(E_{\alpha}^{*}\right)=\left(\gamma_{S}-\gamma_{\alpha}\right) / T_{\alpha}^{* 2}
$$

where $\gamma_{S}=T_{S}^{\prime}\left(E_{\alpha}^{*}\right)$ and $\gamma_{\alpha}=T_{\alpha}^{\prime}\left(E_{\alpha}^{*}\right)$, and the prime denotes differentiation with respect to $E$, ensures the creation of a unimodal probability distribution function, i.e., $P_{\alpha}(E)=e^{-\beta \mathcal{F}_{\alpha}}$. For the unique crossing point $E_{\alpha}^{*}$, between $T_{S}(E)$ and $T_{\alpha}\left(E ; \lambda_{\alpha}\right)$, we demonstrated that $\gamma_{\alpha}\left(E_{\alpha}^{*}\right)<\gamma_{S}\left(E_{\alpha}^{*}\right)$. For linear effective temperatures, $\gamma_{\alpha}$ is a constant equal to $\gamma_{0}$.

Expanding $P_{\alpha}(E)$ to second order at $E_{\alpha}^{*}$ results in

$$
P_{\alpha}\left(E ; \gamma_{\alpha}\right) \approx \exp \left[-\left(E-E_{\alpha}^{*}\right)^{2} / 2 \sigma_{\gamma}\right]
$$

where $\sigma_{\gamma}=T_{\alpha}^{* 2} /\left(\gamma_{S}-\gamma_{\alpha}\right)$, generates a Gaussian PDF centered at $E_{\alpha}^{*}$ with $\gamma_{\alpha}\left(E_{\alpha}^{*}\right)<\gamma_{S}\left(E_{\alpha}^{*}\right)$. 
Fig. 1(a) demonstrates that the linear effective temperatures $T_{\alpha}(E)$ form unique crossing points $E_{\alpha}^{*}$ with statistical temperature $T_{S}(E)$ across the transition region, where $T_{S}(E)$ displays $S$-bends. The parameters in $T_{\alpha}\left(E ; \lambda_{\alpha}\right)=\lambda_{\alpha}+\gamma_{0}\left(E-E_{0}\right)$ are $E_{0}=-43.5 \mathrm{~kJ} \mathrm{~mol}^{-1}, \lambda_{1}=200 \mathrm{~K}, \lambda_{32}=427.6 \mathrm{~K}$, and $\gamma_{0}=-0.101 \mathrm{~mol} \mathrm{~K} \mathrm{~kJ}^{-1}$. The slope of the linear effective temperatures, $\gamma_{0}$, is more negative than the slope of the S-bend part of $T_{S}(E)$, fulfills the condition that $\gamma_{\alpha}\left(E_{\alpha}^{*}\right)<\gamma_{S}\left(E_{\alpha}^{*}\right)$. The resulting GPDFs in Fig. 1(b) are localized around $E_{\alpha}^{*}$ with a Gaussian shape, and naturally bridge between ordered and disordered phases with unimodal energy distributions across the transition region. The results shown in Figs. 1 to 3 are from the system of box length $35.8 \AA$.

In the S-bend region for $T_{S}(E)$, two different energy states can have the same temperature yet have different structures[13]. In order to characterize differing structured states in this nanoconfined water system, we computed the lateral radial distribution function and structure factor.

For the system in Fig. 1, replica 18 and 25 have the same statistical temperature $T_{S}(E)=$ $288.8 \mathrm{~K}$, but replicas 18 and 25 are on the branch of solid and liquid characterized by the lateral radial distribution function and structure factor. (See Fig. 2.) The RDFs of replica 18 and 25 are quite different in terms of the magnitude of the peaks, and the number of peaks, as only three peaks are visible in replica 25 . The structure factor of replica 18 displays a prepeak at $q \approx 2$, a sharp first peak, and a split second peak, in comparison with $S(q)$ for replica 25 . The difference of RDFs and structure factors shows the solid-like and liquid-like characteristics of the configurations in replica 18 and 25, respectively, implying the coexistence of these structurally distinct states in the canonical ensemble.

A sufficiently long simulation with gREM produces the entropy estimate, $S(E)$, by combining results from multiple replicas via ST-WHAM. Once the entropy is determined, canonical thermodynamic properties including internal energy $U(T)$ and heat capacity $C_{v}(T)$, can be calculated as in Eq. (7) and (8). As shown in Fig. 3 (a), the internal energy increases with temperature monotonically across the phase transition region, as a result of reweighting gREM derived caloric curve into the canonical ensemble. The heat capacity $C_{v}$ in canonical ensemble shows a sharp peak at the melting temperature $T_{m}$ in Fig. 3(a). The free energy density, $\mathcal{F}(E, T)=E-T S(E)$, at the melting temperature $T_{m}$ exhibits two local minima at $E_{1}=-38.1$ and $E_{3}=-40.5 \mathrm{~kJ} \mathrm{~mol}^{-1}$ and one local maximum at $E_{2}=-39.5 \mathrm{~kJ} \mathrm{~mol}^{-1}$. The canonical probability density function, $P_{T}(E) \propto e^{-\beta \mathcal{F}(E, T)}$, shows two maxima at $E_{1}$ and $E_{3}$, and a minimum at $E_{2}$. $E_{1}$ and $E_{3}$ correspond to two metastable states and $E_{2}$ corresponds to an unstable state. The bimodal structure in $P_{T}(E)$ demonstrates the intrinsic instability of the canonical ensemble in sampling across the transition region in the vicinity 
of a first-order phase transition [15-18].

We also performed gREM simulations for systems of varying size and used ST-WHAM to compute canonical properties such as the internal energy and heat capacity shown in Fig. 4. The canonical internal energies, $E(T)$, increase monotonically with temperature with a smooth jump in $E(T)$ near the melting temperature. The transitions in the larger systems are more abrupt, as shown by the $E(T)$ curves and the sharp peaks in heat capacity.

Local minima for systems 1 to 6 were produced by the GMIN package using basin-hopping global optimization [55-57]. The structure of system 1 is composed mostly of pentagons. As the system size increases from 2 to 6 , hexagons become the dominant element, with a significant number of pentagons and heptagons.

\section{CONCLUSION}

In this work, we demonstrated the applicability of gREM in simulating the solid-liquid phase transition of bilayer water nanofilms using a monatomic water model. By utilizing a linear effective temperature instead of the canonical temperature, gREM avoids an intrinsic instability of the canonical ensemble in the negative slope region of the statistical temperature accompanying the first-order phase transition. The linear effective temperatures of all replicas were optimized to form unique and stable crossing points with the statistical temperature, resulting in unimodal probability density functions (PDFs) across the phase transition region. Due to the S-bend in the statistical temperature of systems displaying first-order phase transitions, the canonical temperature lacks a one-to-one mapping with energy, and the canonical ensemble may obscure the existence of different states with the same canonical temperature. gREM can resolve these states because it uses energy as the dynamical variable, and samples uniformly from the low energy states to the high energy state with unimodal PDFs. We further examined two states with the same canonical temperature to compare their radial distribution functions and structure factors, which show the solid-like and liquid-like features of the two energy states.

ST-WHAM was used to reweight the gREM simulation results to canonical ensemble so that the thermodynamic variables at canonical temperature can be estimated. The internal energy and heat capacity as a function of temperature were computed and melting temperatures were identified. At the melting temperature, the Helmholtz free energy has double minima and the PDF exhibits a bimodal structure, implying that the transition states are intrinsically unstable in the canonical 
ensemble[13]. The heat capacities and internal energies of systems with varying sizes were computed through gREM simulations and the ST-WHAM reweighting technique. The structures of gREM simulations were optimized using the GMIN package and different crystalline structures are observed for systems with different sizes.

\section{Acknowledgments}

We are grateful to the National Science Foundation (Grant Nos. CHE-1114676) for the generous support of our research.

1 C. Prez, M. T. Muckle, D. P. Zaleski, N. A. Seifert, B. Temelso, G. C. Shields, Z. Kisiel, and B. H. Pate 336, 897 (2012).

2 O. Mishima and H. E. Stanley, Nature (London) 396 (1998).

3 E. B. Moore and V. Molinero, Nature (London) 479 (2011).

4 J. R. Errington, P. G. Debenedetti, and S. Torquato, Phys. Rev. Lett. 89, 215503 (2002).

5 S. Han, M. Y. Choi, P. Kumar, and H. E. Stanley, Nat. Phys. 6 (2010).

6 J. Bai and X. C. Zeng, Proc. Natl. Acad. Sci. U.S.A 109 (2012).

7 N. Kastelowitz, J. C. Johnston, and V. Molinero, J. Chem. Phys. 132 (2010).

8 J. C. Johnston, N. Kastelowitz, and V. Molinero, J. Chem. Phys. 133 (2010).

9 A. A. Bakulin, D. Cringus, P. A. Pieniazek, J. L. Skinner, T. L. C. Jansen, and M. S. Pshenichnikov, J. Phys. Chem. B 117, 15545 (2013).

10 N. Giovambattista, P. J. Rossky, and P. G. Dbenedetti, Phys. Rev. Lett. 5 (2009).

11 P. Kumar, S. V. Buldyrev, F. W. Starr, N. Giovambattista, and H. E. Stanley, Phys. Rev. E 72 (2005).

12 T. Kaneko, J. Bai, K. Yasuoka, A. Mitsutake, and X. C. Zeng, J. Chem. Theo. Comp. 9 (2013).

13 D. J. Wales and R. S. Berry, Phys. Rev. Lett. 73 (1994).

14 F. Calvo, D. J. Wales, J. P. K. Doye, R. S. Berry, P. Labastie, and M. Schmidt, Europhys. Lett. 82, 43003 (2008).

15 J. P. K. Doye and D. J. Wales, J. Chem. Phys. 102, 9673 (1995).

16 D. J. Wales and J. P. K. Doye, J. Chem. Phys. 103, 3061 (1995).

17 R. M. Lynden-Bell and D. J. Wales, J. Chem. Phys. 101, 1460 (1994).

18 D. J. Wales and R. S. Berry, Phys. Rev. Lett. 73, 2875 (1994).

19 J. Kim, T. Keyes, and J. E. Straub, J. Chem. Phys. 132, 224107 (2010).

20 C. J. Geyer and A. Thompson, J. Am. Stat. Assoc. 90, 909 (1995).

21 Y. Sugita and Y. Okamoto, Chem. Phys. Lett. 314, 141 (1999).

22 R. Zhou and B. J. Berne, Proc. Natl. Acad. Sci. U.S.A 99, 12777 (2002).

23 A. E. Garcia and J. N. Onuchic, Proc. Natl. Acad. Sci. U.S.A 100, 13898 (2003).

24 D. Paschek, S. Gnanakarnan, and A. E. Garcia, Proc. Natl. Acad. Sci. U.S.A 102, 6765 (2005).

25 R. Yamamoto and W. Kob, Phys. Rev. E 61, 5473 (2000).

26 E. Flenner and G. Szamel, Phys. Rev. E 73, 061505 (2006).

27 P. A. Frantsuzov and V. A. Mandelshtam, Phys. Rev. E 72, 037102 (2005).

28 P. Poulain, F. Calvo, R. Antoine, M. Broyer, and P. Dugourd, Phys. Rev. E 73, 056704 (2006). 
29 K. Hukushima and K. Nemoto, J. Phys. Sco. Jpn. 65, 1604 (1996).

30 H. Kamberaj and A. van der Vaart, J. Chem. Phys. 127 (2007).

31 U. H. E. Hansmann, Chem. Phys. Lett 281, 140 (1997).

32 A. Mitsutake, Y. Sugita, and Y. Okamoto, Biopolymers 60, 96 (2001).

33 Q. Lu, J. Kim, and J. Straub, J. Phys. Chem. B 116 (2012).

34 Q. Lu, J. Kim, and J. E. Straub, J. Chem. Phys. 138 (2013).

35 W. J. Cho, J. Kim, J. Lee, T. Keyes, J. E. Straub, and K. S. Kim, Phys. Rev. Lett. 112 (2014).

36 J. Kim, T. Keyes, and J. E. Straub, J. Chem. Phys. 135, 061103 (2011).

37 L. G. Rizzi and N. A. Alves, J. Chem. Phys. 135 (2011).

38 M. Church, C. Ferry, and A. E. van Giessen, J. Chem. Phys. 136 (2012).

39 A. M. Ferrenberg and R. H. Swenden, Phys. Rev. Lett. 63, 1195 (1989).

40 E. B. Moore and V. Molinero, J. Chem. Phys. 130, 244505 (2009).

41 L. Le and V. Molinero, J. Phys. Chem. A 115 (2011).

42 E. B. Moore and V. Molinero, Nature 479 (2011).

43 A. Reinhard and J. P. K. Doye, J. Chem. Phys. 136 (2012).

44 Y. Lu, X. Zhang, and M. Chen, J. Phys. Chem. B 117 (2013).

45 J. C. Johnston and V. Molinero, J. Am. Chem. Soc. 134 (2012).

46 R. C. DeMille, T. E. Cheatham, and V. Molinero, J. Phys. Chem. B 115, 132 (2011).

47 A. H. Nguyen and V. Molinero, J. Phys. Chem. B 117 (2013).

48 J. J. Salacuse, A. R. Denton, and P. A. Egelstaff, Phys. Rev. E 53, 2382 (1996).

49 J. N. Herrera, P. T. Cummings, and H. Ruiz-Estrada, Mol. Phys. 96, 835 (1999).

50 Z. Li and H. A. Scheraga, Proc. Natl. Acad. Sci. USA 84, 6611 (1987).

51 D. J. Wales and J. P. K. Doye, J. Phys. Chem. A 101, 5111 (1997).

52 D. J. Wales .

53 J. Nocedal, Mathematics of Computation 35, 773 (1980).

54 D. Liu and J. Nocedal, Math. Program. 45, 503 (1989).

55 Z. Li and H. A. Scheraga, Proc. Natl. Acad. Sci. U. S. A. 84, 6611 (1987).

56 D. J. Wales and J. P. K. Doye, J. Phys. Chem. A 101, 5111 (1997).

57 D. J. Wales and H. A. Scheraga, Science 285, 1368 (1999). 
Figures 

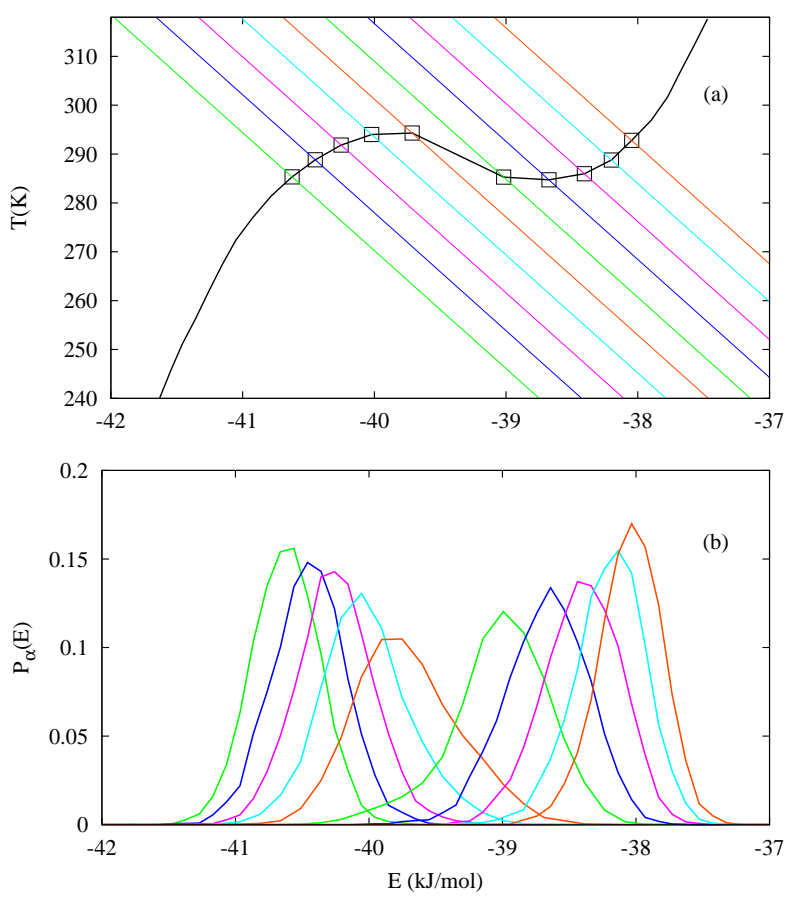

FIG. 1: (a) Effective temperatures $T_{\alpha}(E)$ (a set of parallel lines with negative slope) form unique crossing points (black open squares) with the statistical temperature $T_{S}(E)$ (black curve), (b) generalized probability distributions functions $P_{\alpha}(E)$ of corresponding replicas $\alpha=17,18,19, \ldots, 26$ of the system with size $L x=$ $L y=35.8 \AA$. 

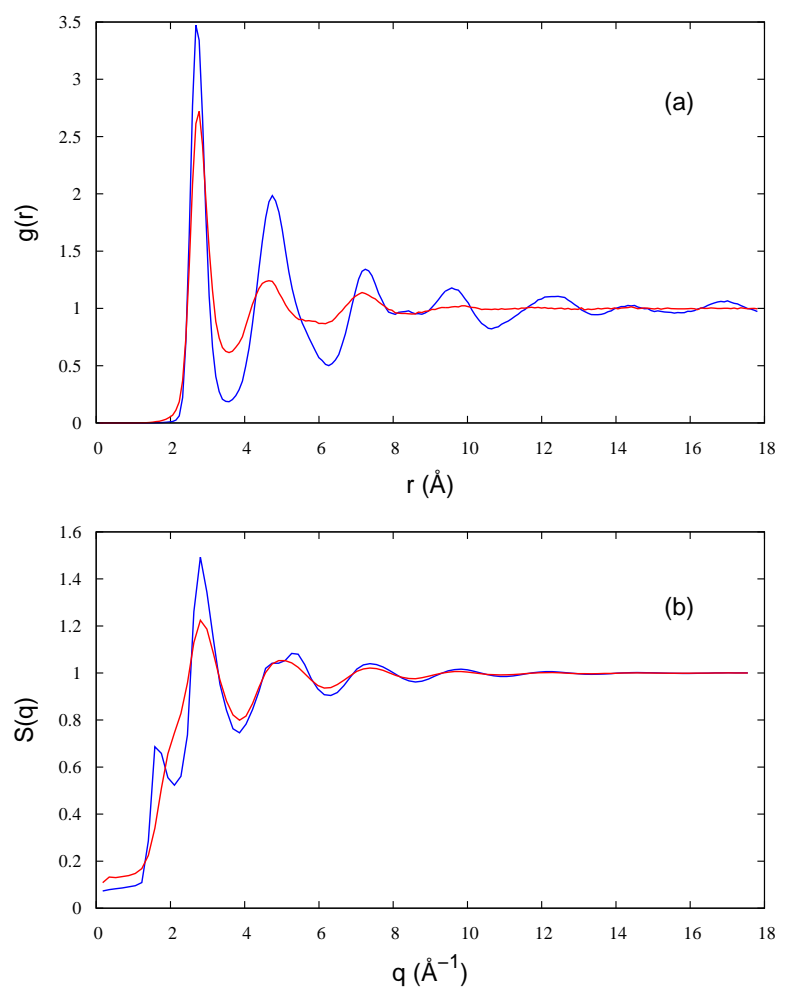

FIG. 2: (a) Lateral radial distribution function $g_{x y}(r)$ and (b) structure factor transformed from $g_{x y}(r)$ of replica 18 (red line) and replica 25 (blue line) of the same system as in Fig.1. 

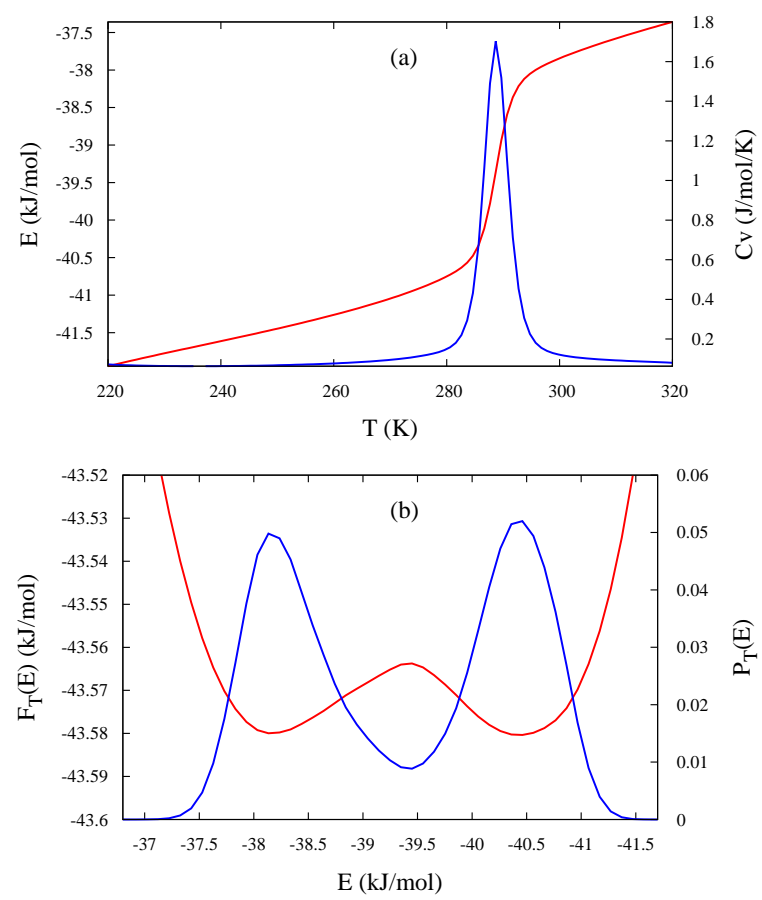

FIG. 3: (a) Energy temperature curve in the canonical ensemble (red line) and molar heat capacity $C_{v}(T)$ (blue line). (b) Probability distribution function $P_{T}(E)$ and free energy $F_{T}(E)$ at the melting temperature $T_{m}$. 

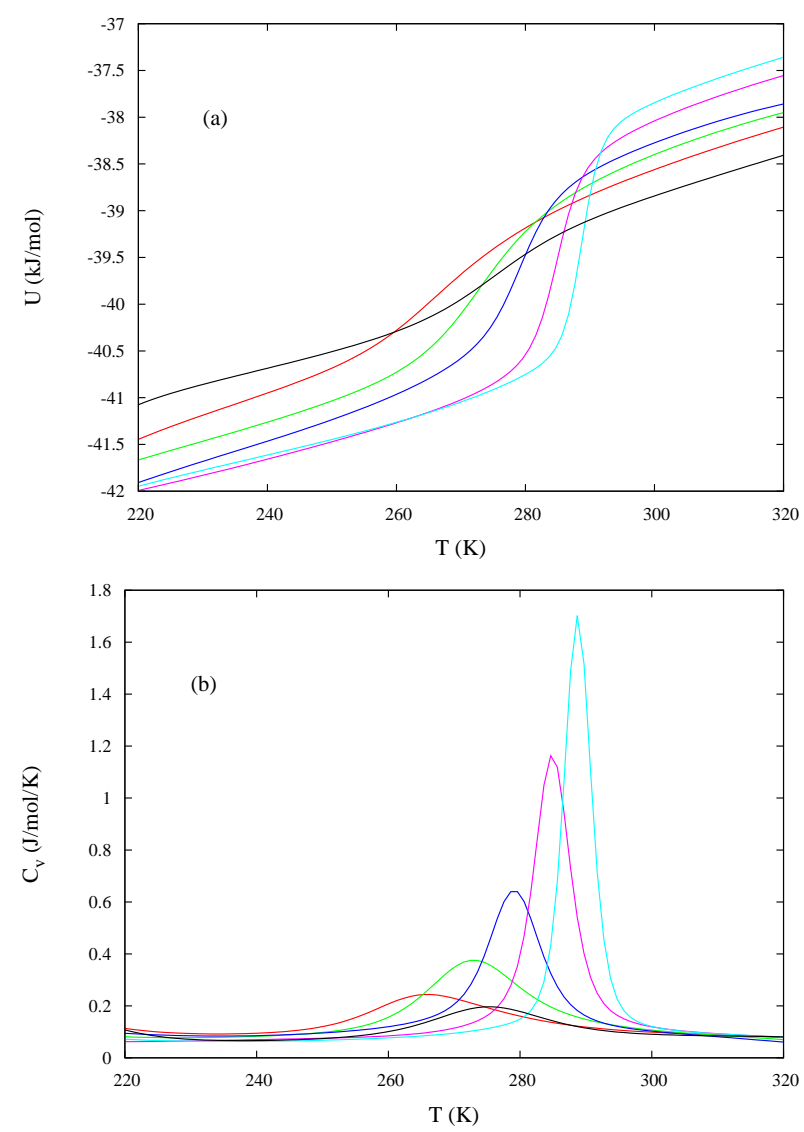

FIG. 4: (a) Energy versus temperature curve, $\mathrm{E}(\mathrm{T})$, and (b) Molar heat capacity, $C_{v}(T)$, of system 1 to 6 . The size of system 1 is $L_{x} \times L_{y}=36.03 \times 31.2 \AA$, while for system 2 to $6, L_{x}=L_{y}=34.5,34.8,35.0,35.5,35.8 \AA$. 


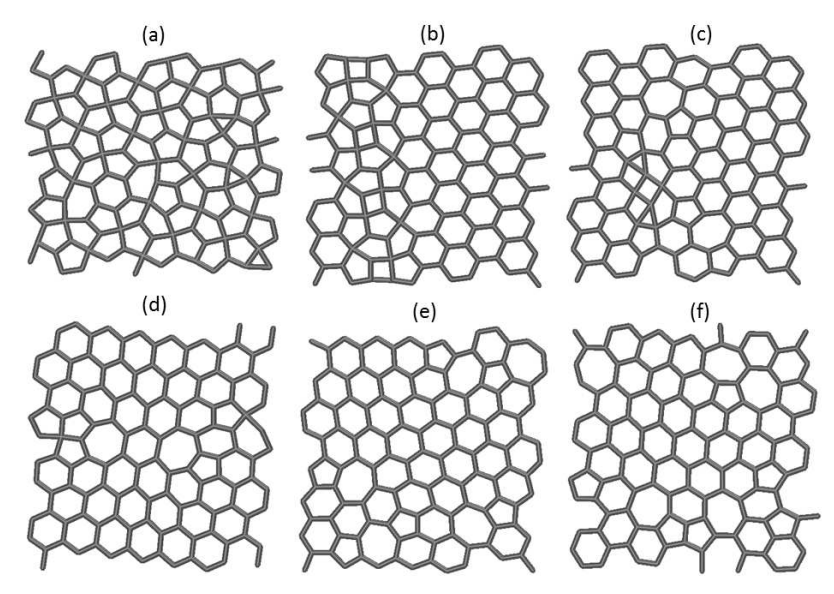

FIG. 5: Minimized structure of systems 1 to 6 produced by the GMIN method. The input structure for each system was the equilibrium structure of the first replica in gREM simulation. 